4. Wakabayashi Y, Umeda K, Yonogi S, Nakamura H, Yamamoto K, Kumeda Y, et al. Staphylococcal food poisoning caused by Staphylococcus argenteus harboring staphylococcal enterotoxin genes. Int J Food Microbiol. 2018;265:23-9. http://dx.doi.org/10.1016/j.ijfoodmicro.2017.10.022

5. Tong SY, Schaumburg F, Ellington MJ, Corander J, Pichon B, Leendertz F, et al. Novel staphylococcal species that form part of a Staphylococcus aureus-related complex: the non-pigmented Staphylococcus argenteus sp. nov. and the non-human primate-associated Staphylococcus schweitzeri sp. nov. Int J Syst Evol Microbiol. 2015;65:15-22. http://dx.doi.org/10.1099/ ijs.0.062752-0

6. McDonald M, Dougall A, Holt D, Huygens F, Oppedisano F, Giffard PM, et al. Use of a single-nucleotide polymorphism genotyping system to demonstrate the unique epidemiology of methicillin-resistant Staphylococcus aureus in remote aboriginal communities. J Clin Microbiol. 2006;44:3720-7. http://dx.doi.org/10.1128/JCM.00836-06

7. Thaipadungpanit J, Amornchai P, Nickerson EK, Wongsuvan G, Wuthiekanun V, Limmathurotsakul D, et al. Clinical and molecular epidemiology of Staphylococcus argenteus infections in Thailand. J Clin Microbiol. 2015;53:1005-8. http://dx.doi.org/10.1128/ JCM.03049-14

8. Aung MS, San T, Aye MM, Mya S, Maw WW, Zan KN, et al. Prevalence and genetic characteristics of Staphylococcus aureus and Staphylococcus argenteus isolates harboring Panton-Valentine leukocidin, enterotoxins, and TSST-1 genes from food handlers in Myanmar. Toxins (Basel). 2017;9:E241. http://dx.doi.org/10.3390/ toxins 9080241

9. Dupieux C, Blondé R, Bouchiat C, Meugnier H, Bes M, Laurent $\mathrm{S}$, et al. Community-acquired infections due to Staphylococcus argenteus lineage isolates harbouring the Panton-Valentine leucocidin, France, 2014. Euro Surveill. 2015;20:21154. http://dx.doi.org/10.2807/1560-7917. ES2015.20.23.21154

10. Chantratita N, Wikraiphat C, Tandhavanant S, Wongsuvan G, Ariyaprasert P, Suntornsut P, et al. Comparison of communityonset Staphylococcus argenteus and Staphylococcus aureus sepsis in Thailand: a prospective multicentre observational study. Clin Microbiol Infect. 2016;22:458.e11-9. http://dx.doi.org/10.1016/ j.cmi.2016.01.008

Address for correspondence: Elisabeth Botelho-Nevers, Infectious Diseases Department, University Hospital of Saint-Etienne, Av Albert Raimond, 42055 Saint-Etienne CEDEX 02, France; email: elisabeth. botelho-nevers@univ-st-etienne.fr

\section{Circulation of Influenza A(H5N8) Virus, Saudi Arabia}

\author{
Hussain Al-Ghadeer, ${ }^{1}$ Daniel K.W. Chu, ${ }^{1}$ \\ Ehab A. Rihan, Ehab M. Abd-Allah, Haogao Gu, \\ Alex W.H. Chin, Ibrahim A. Qasim, Ali Aldoweriej, \\ Sanad S. Alharbi, Marshad A. Al-Aqil, \\ Ali Al-Sahaf, Salah S. Abdel Rahman, \\ Ali H. Aljassem, Ali Abdul-Al, Mohammed R. Aljasir, \\ Yousef M.O. Alhammad, Samy Kasem, \\ Malik Peiris, Ahmed Z.S.A. Zaki, Leo L.M. Poon
}

Author affiliations: Ministry of Environment, Water, and Agriculture, Riyadh, Saudi Arabia (H. Al-Ghadeer, E.A. Rihan, E.M. Abd-Allah, I.A. Qasim, A. Aldoweriej, S.S. Alharbi, M.A. Al-Aqil, A. Al-Sahaf, S.S. Abdel Rahman, A.H. Aljassem, A. Abdul-Al, M.R. Aljasir, Y.M.O. Alhammad, S. Kasem, A.Z.S.A. Zaki); University of Hong Kong, Hong Kong (D.K.W. Chu, H. Gu, A.W.H. Chin, M. Peiris, L.L.M. Poon); Kafr El-Sheikh University, Kafr El-Sheikh, Egypt (S. Kasem)

\section{DOI: https://doi.org/10.3201/eid2410.180846}

Highly pathogenic avian influenza $\mathrm{A}(\mathrm{H} 5 \mathrm{~N} 8)$ viruses have been detected in several continents. However, limited viral sequence data are available from countries in the Middle East. We report full-genome analyses of highly pathogenic H5N8 viruses recently detected in different provinces in Saudi Arabia.

$\mathrm{O}^{\prime \prime}$ necember 19, 2017, a high number of dead birds from various species was reported in a live bird market in Riyadh, Saudi Arabia, by the Department of Animal Resources Services, Ministry of Environment, Water, and Agriculture. Oropharyngeal and cloacal swab samples were collected from affected birds and investigated for highly pathogenic avian influenza (HPAI) viruses in Riyadh Veterinary Diagnostic Laboratory using reverse transcription PCR (RT-PCR) (1). These tests detected HPAI A(H5N8) virus. After this index outbreak, HPAI was reported in adjacent provinces. Surveillance studies were performed in all provinces ( $\geq 1$ major poultry market and 10 backyard farms per province) to estimate disease prevalence. As of May 2018, a total of 7,273 birds had been investigated; 805 were positive for $\mathrm{H} 5 \mathrm{~N} 8$, which was detected in 7 provinces (Riyadh, Eastern, Al-Qasim, Makkah, Al-Madinah, Asir, and Jizan). The highest number of positive results was reported in Riyadh (693 samples), in which different commercial poultry farms (22 farms for laying hens, 2 for broiler breeders, and 1 for quail) were affected. A contingency plan, based on a stamping-out policy, was implemented to control the disease. More than 8.8 million birds were depopulated.

${ }^{1}$ These authors contributed equally to this article. 
Positive clinical specimens $(\mathrm{N}=14)$ collected from different settings, different provinces, different avian species or a combination were sent to a World Health Organization H5 reference laboratory in Hong Kong for confirmation. All samples tested positive for membrane protein (M) and hemagglutinin (HA) subtype H5 genes by RT-PCR (online Technical Appendix Table 1, https://wwwnc.cdc.gov/EID/ article/24/10/18-0846-Techapp.pdf). Samples that had a cycle threshold value $<29$ in the $\mathrm{M}$ gene assay also tested positive for N8 by RT-PCR. Ten of these samples were positive for virus isolation in embryonated chicken eggs and were associated with death of the chicken embryos by day 3 postinoculation.

We amplified viral RNA extracted from the clinical specimens and virus isolates using a multisegment RT-PCR approach for full-genome amplification (2). We subjected the RT-PCR products to next-generation sequencing on an Illumina MiSeq (PE300) platform (Illumina, San Diego, CA, USA). We edited the deduced consensus sequences (average sequence coverage $>10,000 \times$ ) using BioEdit (https://www.mbio.ncsu.edu/BioEdit/bioedit.html) and analyzed them phylogenetically using MEGA7 (https:// www.megasoftware.net) (GISAID accession nos. for reference sequences, EPI1215422-EPI1215461, EPI1215137EPI1215184; http://platform.gisaid.org).

The deduced sequences revealed that $\mathrm{H} 5 \mathrm{~N} 8$ viruses ( $\mathrm{n}$ =11) from different sites in Saudi Arabia are almost identical (sequence identity $>99.7 \%$ ), indicating a common origin for this outbreak. Phylogenetic analyses of HA sequences showed that they belong to clade 2.3.4.4 group B (Figure) (3). Polymerase acidic protein (PA), HA, nucleoprotein (NP), neuraminidase (NA), M, and nonstructural protein (NS) segments were genetically similar to those derived from recent group B H5N8 viruses (online Technical Appendix Table 2, Figure 1). No genetic markers associated with mammalian host adaptation, $\alpha 2,6$ receptorbinding specificity, or antimicrobial drug resistance were detected (data not shown) (4). The gene constellation of PA, HA, NP, NA, M, and NS segments of these H5N8 viruses is similar to those of some $\mathrm{H} 5 \mathrm{~N} 8$ viruses detected in wild migratory birds from different geographic areas (e.g., A/Anser_cygnoides/Hubei/FW44/2016 and A/greenwinged teal/Egypt/877/2016) $(4,5)$. The polymerase basic protein (PB) 1 and 2 segments of these viruses are similar to those of HPAI H5N5 viruses detected in the Far East (e.g., A/environment/Kamchatka/18/2016) and Europe (e.g., A/swan/Germany-SN/R10645/2016) (online Technical Appendix Figure 1). H5N5 viruses of this lineage were previously proposed to be reassortants of an $\mathrm{H} 5 \mathrm{~N} 8$ virus (6), with the PB1 and PB2 segments derived from an $\mathrm{H} 10$ virus (A/duck/Mongolia/245/2015-like virus) and the PA, HA, M, and NS segments derived from a H5N8 virus. Our results agree with previous observations that
H5N8 viruses of this lineage continue to evolve and reassort with other influenza virus subtypes in migratory bird populations $(7,8)$.

The studied samples were collected from multiple avian species in different settings from 3 provinces (online Technical Appendix Table 1). Of 986 samples from poultry holding sites, $182(18.5 \%)$ tested positive for $\mathrm{H} 5 \mathrm{~N} 8$ virus. The transmission pathway of $\mathrm{H} 5 \mathrm{~N} 8$ virus in Saudi Arabia is being investigated. Molecular dating analyses suggest that the most recent common ancestor of these H5N8 viruses emerged in this country in September 2017 (online Technical Appendix Figure 2). The potential roles of wild birds, backyard poultry practices, poultry trading, and other human activities in dissemination of these viruses are yet to be determined. However, our results suggest wide circulation of $\mathrm{H} 5 \mathrm{~N} 8$ viruses caused by a single introduction.

Recently, outbreaks of H5N8 viruses were reported in the Middle East (Israel, Iran, Iraq, and Kuwait) (1). However, with the exception of a few HA sequences $(n=12)$, no other H5N8 viral sequences from this region are available in major sequence databases, which has hampered the investigation of $\mathrm{H} 5 \mathrm{~N} 8$ viruses in this region. Multiple introductions of $\mathrm{H} 5 \mathrm{~N} 8$ viruses with different gene constellations have been reported in Egypt $(9,10)$, but their genetic relationship to H5N8 viruses detected in other countries in the Middle East is not clear. Further surveillance using fullgenome analyses is urgently needed to identify major risk factors for HPAI H5N8 viruses in the Middle East.

\section{Acknowledgments}

The role of the Deputy Ministership for Animal Resources at the Ministry of Environment, Water, and Agriculture (MEWA) of Saudi Arabia in quick reporting and confirmation of the HPAI outbreak is highly appreciated. The authors acknowledge all the teams that supported the H5N8 investigation from MEWA. We acknowledge the authors and the originating and submitting laboratories of the sequences from GISAID's EpiFlu Database (http://platform.gisaid.org) with which this analysis was carried out.

The work conducted in Hong Kong was supported in part by the National Institute of Allergy and Infectious Diseases, National Institutes of Health (contract no. N272201400006C), Themebased Research Scheme from Research Grants Council of the Hong Kong Special Administrative Region, China (project no. T11-705/14N).

\section{About the Author}

Dr. Al-Ghadeer is a virologist at Riyadh Veterinary Diagnostic Laboratory, Ministry of Environment, Water, and Agriculture, Riyadh, Saudi Arabia. His main research interest is molecular diagnosis of viral diseases and viral genome sequencing. 


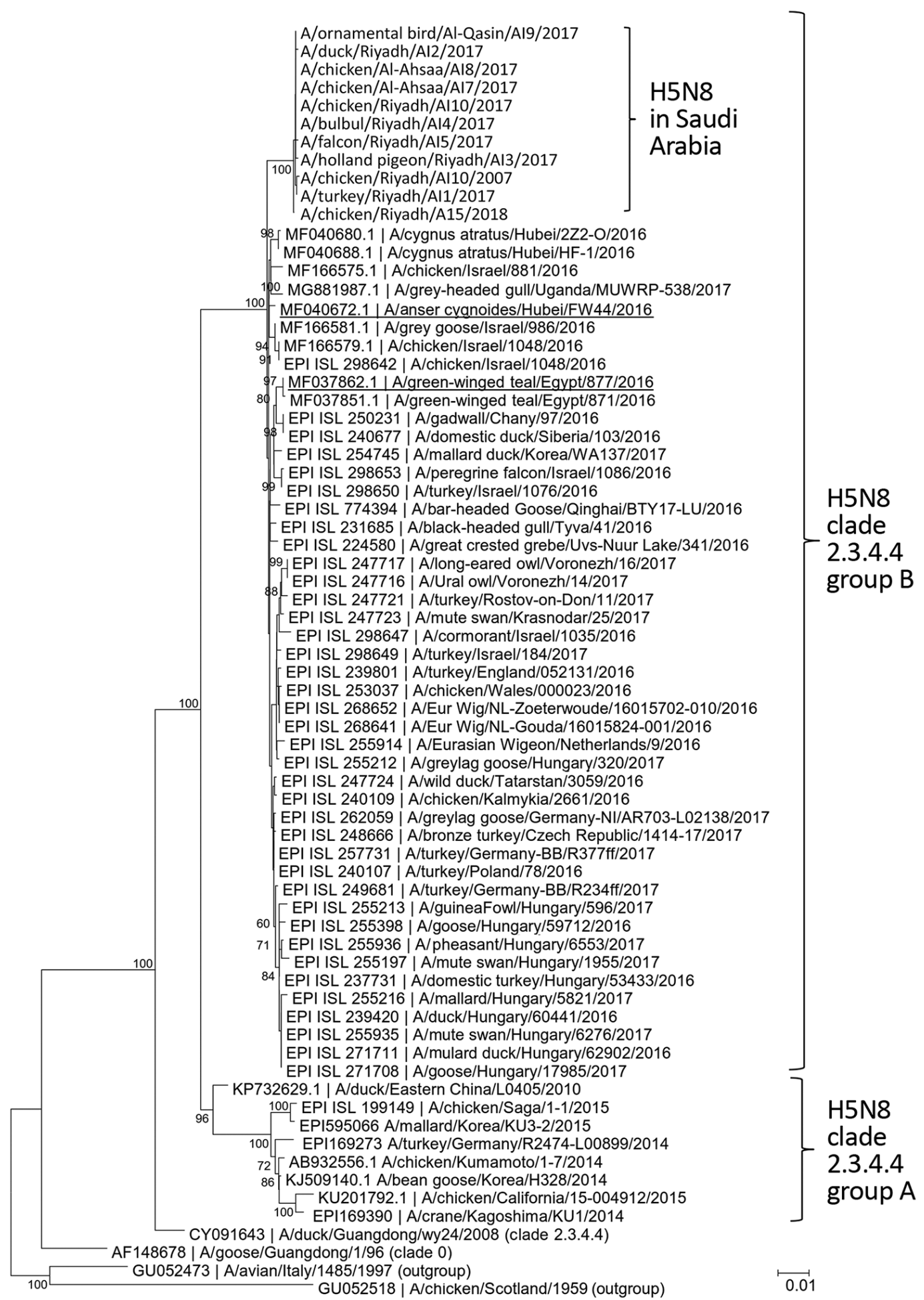

Figure. Phylogenetic analysis of hemagglutinin sequences of influenza $A(H 5 N 8)$ viruses detected in oropharyngeal and cloacal swab samples from birds in Saudi Arabia. Aligned sequences were analyzed in MEGA7 (http://www.megasoftware.net). We constructed the phylogenetic tree using the neighbor-joining method. Representative viral sequences and viral sequences that are highly similar to those reported in this study were included in the analysis. H5N8 viruses reported in this study are labeled. Bootstrap values $\geq 60 \%$ are shown. Representative viruses sharing a similar gene constellation as the H5N8 viruses found in Saudi Arabia are underlined (see text for details). Virus isolate numbers (EPI ISL) in GISAID (http://platform.gisaid.org) or gene accession numbers in GenBank for corresponding viral sequences are provided. Scale bar indicates estimated genetic distance. 


\section{References}

1. World Organisation for Animal Health. Update on avian influenza in animals (types H5 and H7) [cited 2018 May 19]. http://www.oie.int/en/animal-health-in-the-world/update-onavian-influenza/

2. Zhou B, Donnelly ME, Scholes DT, St. George K, Hatta M, Kawaoka Y, et al. Single-reaction genomic amplification accelerates sequencing and vaccine production for classical and swine origin human influenza A viruses. J Virol. 2009;83:1030913. http://dx.doi.org/10.1128/JVI.01109-09

3. El-Shesheny R, Barman S, Feeroz MM, Hasan MK, Jones-Engel L, Franks J, et al. Genesis of influenza A(H5N8) viruses. Emerg Infect Dis. 2017;23:1368-71. http://dx.doi.org/10.3201/ eid2308.170143

4. Ma L, Jin T, Wang H, Liu H, Wang R, Li Y, et al. Two reassortant types of highly pathogenic H5N8 avian influenza virus from wild birds in Central China in 2016. Emerg Microbes Infect. 2018;7:14. http://dx.doi.org/10.1038/s41426-017-0012-y

5. Kandeil A, Kayed A, Moatasim Y, Webby RJ, McKenzie PP, Kayali G, et al. Genetic characterization of highly pathogenic avian influenza A H5N8 viruses isolated from wild birds in Egypt. J Gen Virol. 2017;98:1573-86. http://dx.doi.org/10.1099/ jgv.0.000847

6. Fusaro A, Monne I, Mulatti P, Zecchin B, Bonfanti L, Ormelli S, et al. Genetic diversity of highly pathogenic avian influenza A(H5N8/H5N5) viruses in Italy, 2016-17. Emerg Infect Dis. 2017;23:1543-7. http://dx.doi.org/10.3201/eid2309.170539

7. Pohlmann A, Starick E, Grund C, Höper D, Strebelow G, Globig A, et al. Swarm incursions of reassortants of highly pathogenic avian influenza virus strains $\mathrm{H} 5 \mathrm{~N} 8$ and $\mathrm{H} 5 \mathrm{~N} 5$, clade 2.3.4.4b, Germany, winter 2016/17. Sci Rep. 2018;8:15. http://dx.doi.org/10.1038/ s41598-017-16936-8

8. Global Consortium for H5N8 and Related Influenza Viruses. Role for migratory wild birds in the global spread of avian influenza H5N8. Science. 2016;354:213-7. http://dx.doi.org/10.1126/science. aaf8852

9. Salaheldin AH, El-Hamid HSA, Elbestawy AR, Veits J, Hafez HM, Mettenleiter TC, et al. Multiple introductions of influenza A(H5N8) virus into poultry, Egypt, 2017. Emerg Infect Dis. 2018;24:943-6. http://dx.doi.org/10.3201/eid2405.171935

10. Yehia N, Naguib MM, Li R, Hagag N, El-Husseiny M, Mosaad Z, et al. Multiple introductions of reassorted highly pathogenic avian influenza viruses (H5N8) clade 2.3.4.4b causing outbreaks in wild birds and poultry in Egypt. Infect Genet Evol. 2018;58:56-65. http://dx.doi.org/10.1016/j.meegid.2017.12.011

Address for correspondence: Leo L.M. Poon, the University of Hong Kong School of Public Health, LKS Faculty of Medicine, 5/F William MW Mong Block, Bldg 21, Sassoon Road, Hong Kong, China; email: 1lmpoon@hku.hk; or Hussain Al-Ghadeer, Riyadh Veterinary Diagnostic Laboratory, General Administration of Laboratories, Ministry of Environment, Water, and Agriculture, Riyadh 11454, Saudi Arabia; email: E23315@mewa.gov.sa

\section{Severe Respiratory Illness Outbreak Associated with Human Coronavirus NL63 in a Long-Term Care Facility}

\author{
Julie Hand, Erica Billig Rose, Andrea Salinas, \\ Xiaoyan Lu, Senthilkumar K. Sakthivel, \\ Eileen Schneider, John T. Watson
}

Author affiliations: Louisiana Department of Health, Baton Rouge, Louisiana, USA (J. Hand, A. Salinas); Centers for Disease Control and Prevention, Atlanta, Georgia, USA (E.B. Rose, X. Lu, S.K. Sakthivel, E. Schneider, J.T. Watson)

DOI: https://doi.org/10.3201/eid2410.180862

We describe an outbreak of severe respiratory illness associated with human coronavirus NL63 in a long-term care facility in Louisiana in November 2017. Six of 20 case-patients were hospitalized with pneumonia, and 3 of 20 died. Clinicians should consider human coronavirus NL63 for patients in similar settings with respiratory disease.

$\mathrm{H}$ uman coronaviruses (HCoVs) OC43, 229E, NL63, and HKU1 are frequently associated with upper respiratory tract infection but can also cause lower respiratory tract infections (LRTIs), such as pneumonia or bronchitis. Transmission of these viruses primarily occurs through respiratory droplets and indirect contact with secretions from infected persons. Signs and symptoms of illness often include runny nose, headache, cough, sore throat, and fever. LRTI occurs less frequently, but young children, older adults, and persons who are immunosuppressed appear to be at higher risk for these types of infections (1-3).

A wide range of respiratory viruses are known to circulate in long-term care facilities (LTCFs) and contribute to respiratory illness in the residents who live in them (4). Although outbreaks of HCoV-OC43 have been described among elderly populations in long-term care settings (5), outbreaks of severe respiratory illness associated with HCoV-NL63 have not, to our knowledge, been documented in LTCF settings.

On November 15, 2017, the Louisiana Department of Health (Baton Rouge, Louisiana, USA) was notified of a possible outbreak of severe respiratory illness by a representative of an LTCF that provides nursing home care and short-term rehabilitation services to 130 residents. At the time of notification, the facility reported 11 residents with chest radiograph-confirmed pneumonia. For this investigation, we defined a case-patient as any LTCF resident with respiratory tract symptoms of new onset in November 2017, and we considered LRTI diagnoses that were based 